\title{
A propos du Symposium sur la cavitation (Londres-Septembre 1955)
}

\section{The Cavitation Symposium (London September 1955)}

\author{
PAR J. DUPORT
}

L'exposé de $M$. Duport n'a nullement la prétention de résumer les travanx du Symposium sur la cavitation organisé en septembre 1955 par le "National Physical Laboratory" à Londres. L'auteur circonscrit son sujet a l'examen critique des règles de similitude généralement appliquées pour l'étude du seulil de cavitation des turbines hydranliques.

Les phénomènes par lesquels se manifeste la cavitation : bruit, vibrations, érosion, chute de rendement s'accentuent brutalement si, au-delà d'un régime limite, on aggrave progressivement les conditions de leur apparition. On peut donc valablement parler d'un seuil de cavitation. En réalité, cette notion de seuil est moins nette qu'il ne parait d'abord : le senil et les. lois de similitude correspondantes dépendent, dans une certaine mesure, de celle des manifestations par laquelle on prétend déceler le début de la cavitation.

Selon l'anteur, il ne semble pas que l'ingénieur ayant mission de concevoir on d'exploiter les turbines hydrauliques, ait $\dot{a}$ se préoccuper, comme le physicien, de l'apparition des toutes premieres bulles de vapeur.

Dans la mesure oì l'on ne s'attache qu'à l'étude des manifestations plus sensibles, il semble que lon puisse se contenter de la similitude des pressions, ce qui conduit à l'asage combiné des lois de Thoma et de Froude.

M. Duport en voit la preuve surtout dans la cohérence des résultats obtenus par cette méthode, lorsque l'on compare le comportement d'un modele et d'un prototype on lorsqu'on synthétise les résultats de l'expérience à l'échelle industrielle.

L'analyse des études présentées à Londres confirme d'ailleurs que si la similitude des pressions néglige certains factears, tels que fluctuations turbulentes, vitesse d'expansion et de collapsus des bulles, etc., it semble bien qu'il ne s'agisse lù que de phénomènes secondaires, au point de vue de l'ingénieur.

Ceci, tontefois, n'est exact qu'au prix de certaines précautions indispensables : maintien d'une certaine quantité d'air dissons dans l'eau, échelles suffisamment grandes des modèles par exemple.

I'autenr conclut en montrant que si la connaissance en matiexe de cavitation a notablement progressé recemment, il reste encore beancoup it trouver. Cette relative ignorance doit inciter les constructenrs et les maitres d'ouvre a une certaine prudence dans le choix des tracés et des calages des turbines hydrauliques.
Mr. Duport's article is not intended to be a resume of the work of the Symposium on Cavitation which was held in September 1955 in London under the auspices of the National physical Laboratory. The author confines himself to a critical examination of the laws of similitude usually invoked when studying the cabitation threshold of water turbines.

The phenomena which betray cavitation, such as noise, vibration, erosion and falls in elficiency, are sharply accentuated if, after a lintiting state is reached, the conditions under which they appear are progressively worsened. It is therefore quite legitimate to speak of a cavitation threshold.

In fact, the concept of a threshold is not so clearly defined as it would seem to be on first sight. The threshold and the corresponding laws of similitude are to a certain extent dependent upon which criterion is used to detect the onset of cavitation.

It is the anthor's contention that engineers concerned with designing and operating water turbines should not share the physicist's preoccupation with the very first vapour bubbles that appear.

If we confine ourselves to studying the more easily discernible phenomena it wonld seem that the pressure similitude is sufficient and leads to a combined use of Thoma's and Froude's laws.

In Mr. Duport's opinion this is demonstrated above all by the coherence of results obtained using this method when the behaviour of a model is compared with that of a protolype or when experimental results are used to give indications of industrial qualities.

Furthermore an analysis of the papers presented in London indicates that if the pressure similitude neglects certain factors such as tarbulent fluctuations and the rate at which bubbles expand and collapse, it would seem that these phenomena are only of secondary importance to the engineer.

This remains true only if certain essential precautions are taken. For example a certain quantity of air must be kept dissolved in the water and madels must be built to a sufficientty large scale.

The anthor concludes' by showing that althongh our knowledge of cavitation has become much greater recently there is still much to be found out. This relative lack of knowledge ought to cause manufacturers and contracting anthorities to exercise a certain amount of prudence when determining hydraulic profiles for tur bines and when positioning runners. 


\section{INTRODUCTION}

Au cours du Symposium sur la cavitation organisé du 14 au 17 septembre par le National Physical Laboratory à Teddington, plus de vingt communications ont été présentées. Les discussions et commentaires ont souvent été d'une importance et d'un intérêt comparables à celles des conférences elles-mêmes; aussi n'ai-je nullement la prétention, dans le peu de temps qui m'est alloué, de résumer ces travaux qui, d'ailleurs, feront certainement l'objet d'une publication intégrale.

Il m'a semblé plus sage de me contenter de présenter certaines réflexions inspirées par les exposés du Symposium et ayant trait à l'étude de la cavitation dans les turbines hydrauliques. Nous nous limiterons d'ailleurs à l'examen de quelques problèmes de similitude dont les solutions peuvent être recherchées d̀ la lumière des travaux présentés ou évoqués à Londres.

Dès qu'on parle de similitude on pent penser qu'il s'agit des règles de l'expérimentation sur modèle réduit et de la transposition des performances du modèle à l'échelle industrielle. Sans doute, mais pas uniquement: la comparaison des résultats observés sur les grandes machines, la synthétisation des connaissances acquises à l'échelle industrielle supposent déjà le recours à une certaine similitude: le sigma de Thoma, par exemple, qui exprime la loi fondamentale de similitude de cavitation, a été imaginé par son auteur tout autant pour systématiser les renseignements statistiques sur les turbines industrielles que pour interpréter les résultats d'essais sur modèle de cavitation.

Cette loi de Thoma à laquelle je viens de faire allusion, on s'est demandé à Londres si elle suffit à traduire tous les aspects de la cavitation et même s'il ne vaudrait pas mieux parfois l'abandonner pour se rendre Jibre d'ajuster d'autres paramètres (car les ingénieurs qui jouent du modèle réduit n'arrivent jamais à ajuster beaucoup de parametres a la fois: Thoma, Froude, Reynolds et Weber par exemple).

Le problème devient moins épineux, à mon avis, si l'on veut bien considérer que les lois de similitude, comme toutes les lois physiques peut-être, ne peuvent prétendre refléter que partiellement la réalité, ne rendent compte fidèlement que de certains aspects des phénomènes naturels tonjours complexes.

\section{I. - LE OU LES SEUILS DE CAVITATION}

Quelles sont les manifestations de la cavitalion qui intéressent le turbinier, quelles sont les règles de similitude les mieux adaptées à leur représentation ? C'est ce que nous allons essayer de dégager des travaux présentés à Londres.

Pour l'ingénieur qui projette, construit ou exploite des turbines hydrauliques, la cavitation c'est d'abord du bruit, parfois même beaucoup de bruit. Ce n'est déjà pas très agréable d'entendre dans une machine sensée véhiculer sagement de l'eau, des roulements de cailloux, des coups de pistolets ou de canons, mais cela devient plus angoissant si le fond de la turbine et la butée se mettent à avoir des soubresauts qui incitent les spectaleurs à chercher la sortie. la plus proche.

Moins angoissante mais tout aussi grave est la baisse de rendement ou de puissance qui cause au constructeur bien des ennuis et à l'exploitant des pertes qui peuvent être substantielles.

L'érosion des rones, aprés tout cela, nous. paraitrait un mal bénin si nous ne savions les répercussions que, trop rapides ou trop accentuées, elles peuvent avoir sur le rendement d'une installation, et sur le coût de l'exploitation.

Ces effets variés, on le sait maintenant, proviennent d'une seule et même cause : une sorte d'ébullition sous vide provoquée par les dépressions dynamiques que l'eau subit en traversant la turbine. Ces phénomènes s'accentuent très rapidement, brutalement même, lorsque au-delà d'un certain seuil on aggrave graduellement les conditions de leur apparition: une minime augmentation d'ouverture, une baisse légère de niveau aval à partir d'un régime exempl da cavitation peuvent faire natre des tumultes jugés intolérables ou un effondrement du wattmètre, ou les deux ensemble.

Parce que le comportement des machines audelà de ce seuil paraît à la fois si précaire et si rebelle à l'analyse, les ingénieurs se sont d'abord attachés à caractériser ces conditions 
limites pour éviter de les dépasser. C'est encoro aujourd'hui leur préoccupations majeure et l'on peut dire que la similitude de cavitation qui intéresse le plus le turbinier c'est la similitude du seuil de cavitation.

Cette préoccupation cependant n'est pas exclusive. Comme le rappelle par exemple $\mathrm{M}$. Numachi dans sa communication, linfluence de la cavitation sur la vitesse d'emballement juslifie qu'on se préoccupe des lois qui la régissent, or a l'emballement on a pratiquement toujours dépassé le seuil de cavitation.

L'étude du seuil de cavitation oblige d'ailleurs à analyser ce qui se passe plus ou moins loin au-delà, car cette notion de seuil est en réalité moins nette qu'il n'y paraît d'abord, et il convient de s'entendre sur ce qu'on appellera le commencement de la cavitation.

Pour le physicien, le début de cavitation c'est le moment où, parmi les microscopiques noyaux de gaz non dissous, ou de vapeur, pré-existant au sein du Jiquide, certains commencent sous l'effet de dépressions locales à se dilater puis à se résorber brutalement en arrivant dans les zones de pression accrue.

Cetle façon de définir le début de cavitation est-elle bien celle qui correspond aux préoccupations de l'ingénieur et plus particulièrement du turbinier? Nous ne le pensons pas, car l'existence d'un petit nombre de bulles de vapeur se dilatant, puis se résorbant dans la turbine n'est certainement pas suffisante pour causer des dommages sensibles aux parois métalliques ou aux performances de la machine.

Il est bien probable que si l'on examinait avec l'oil du physicien toutes les turbines existantes, toutes, y compris celles qui sont actuellement réputées exemptes de cavitation, paraîtraient plus ou moins atteintes; tout comme les patients du docteur Knock n'étaient, bien portants, que des malades qui s'ignoraient.

Pour nous, modestes patriciens qui aidons les turbines à naitre, ou qui plus tard les soignons au cours de leur longue vie, nous nons attachons seulement à leur ćviter les grosses maladies, et à les maintenir aussi longtemps que possible en pleine vigueur.

Les «seuils de cavitation que, de ce point de vue on pourrait défnir, pourrait concerner :

- Soit la limite an-delà de laquelle les performances (rendement, puissance) commencent à être altérées ;

- Soil l'état à partir duquel les vibrations et fatigues infligées à la machine deviennent dangereuses ;

- Soit enfin les conditions à respecter pour que l'érosion ne se développe pas à une allure ruineuse pour l'exploitation.

Les deux derniers critères sont rarement pris en considération, du moins sous une forme précise; peut-être parce que leur définition est quelque peu subjective, certainement aussi parce que les paramètres qui les déterminent sont encore mal connus.

Le senil de chute de rendement ou de puissance, est le plus couramment utilisé, en particulier lors d'essais de cavitation sur modèle réduit.

\section{II. - FACTEURS INFLUENÇANT LE DÉBUT DE CAVITATION}

\section{1) Au sens du physicien.}

Les conditions d'apparition des premières bulles de vapeur sont certainement très complexes. M. Eisenberg dans le magnifique exposé de synthèse par lequel il a inauguré les travaux du Symposium, a retracé les grandes lignes de la recherche récente en cette matière.

Il semble résulter des travaux sur ce sujet que l'écart entre la pression locale moyenne et la tension de vapeur que l'on prend en compte dans la similitude de Thoma, n'est pas seul en cause :

a) L'eau peut parfois supporter, sans caviter, des dépressions assez importantes,

- Soit par suite de la pénurie en noyaux préexistants (pénurie sans doute liée, mais d'une facon mal connue, à la teneur en air ou gaz dissous),

- Soit par l'application très brève de la pression négative.

b) L'équilibre dynamique des bulles peut ne pas correspondre à une pression égale à la tension de vapeur : la tension superficielle, la viscosité, la compressibilité de l'eau, celle des gaz contenus dans la bulle, et les phénomènes thermo-dynamiques qui y sont liés; jouent un rôle certain dans la dilatation et le collapsus.

c) La pression locale instantanée peut différer de celle dont les lois de Thoma et Froude expriment la similitude par suite des phénomènes de couche limite et des fluctuations turbulentes. 
d) Les gradients de pression peuvent aussi avoir leur importance, et certains auteurs ont pu suggérer une sorte d'échelle de temps où intervient la vitesse de déplacement de la bulle entraînée par le courant dans un champ de pression variable, et le déroulement du processus de dilatation et de collapsus.

e) Enfin les auteurs de plusieurs communications ont fait remarquer que pour définir convenablement l'apparition des premières bulles, il faut avoir précisé la façon dont on décèle leur présence. Les uns se contentent de regarder à l'œil nu, les autres utilisent des moyens plus ou moins perfectionnés de photographie et d'agrandissement, les autres écoutent d'abord avec un stétoscope, puis avec un microphone dont ils enregistrent les indications. Il semble que le procédé le plus fin corresponde à l'observation acoustique.

\section{2) Au sens du turbinier.}

On peut s'attendre à ce que, au moment où le rendement et la puissance commencent à être altérés, la cavitation soit déjà quelque peu développée, car elle doit s'étendre nécessairement sur une part non négligeable de la surface des aubes motrices; c'est ce que montre très nettement la photographie ci-jointe qui correspond au sigma critique de chute de rendement d'une turbine Kaplan et oì l'on voit nettement une poche de cavitation suffisamment grande pour être tout-à-fait visible à l'œil nu.

Nous allons maintenant examiner quelles règles de similitude doivent, à notre sens, être prises en compte pour étudier ce stade critique du développement de la cavitation.

Bien entendu, les lois de similitude particulières aux phénomènes de cavitation doivent être utilisées concurremment avec les règles habituelles : similitude géométrique, similitude des triangles de vitesse (connue en France sous le nom de similitude de Combes-Rateau).

Parmi les facteurs affectant le début de cavilation, nous voudrions régler tout d'abord la. question des noyaux pré-existants dans l'eau et de l'éventuel retard à la cavitation. Nous ne pensons pas que les conditions rendant possible ce retard puissent se produire dans les eaux utilisées dans les turbines industrielles: la teneur en air dissous, la présence d'impuretés qui entraînent de petites bulles d'air ou de gaz, tout ceci nous laisse penser qu'on n'a pratiquement jamais de retard à la cavitation dans les machines industrielles.

Par contre, on ne peut pas le dire aussi nettement des eaux utilisées dans les laboratoires, et des précautions doivent certainement être prises à ce sujet. Les résorbeurs d'air, tels qu'on peut maintenant les étudier et les construire grâce aux recherches et aux mises au point du Professeur Knapp, apportent à ce problème une solution excellente.

Nous étant ainsi prémunis contre le risque du retard à la cavitation, pouvons-nous nous fier à la similitude de Thoma, et devons-nous, comme le professeur Thoma l'avait lui-même préconisé, associer l'égalité des signa à celle des nombres de Froude.

Sur le premier point nous pensons pouvoir répondre par l'affirmative, nous basant:

a) D'une part sur le fait que le sigma de Thoma a été, universellement utilisé semblet-il avec succès, depuis plus de vingt. ans.

b) D'autre part sur de nombreuses expériences, comme celles rapportées par M. Numachi, où effectuées par nous-mêmes et au cours desquelles le sigma a paru ètre nettement caractéristique, sans effet marqué d'effet de couche limite ou de turbulence locale.

Nous voudrions évoquer, cependant, deux aspects de l'effet d'échelle qui, à notre sens, ne peuvent être négligés quand on essaie une turbine à la cavitation sur modèle réduil (ou mème quand on compare deux machines de tailles différentes): les fluctuations de pression dans une turbine n'ont pas seulement pour origine la turbulence locale, il en existe d'autres ayant pour origine une turbulence à beaucoup plus grande échelle, par exemple des lâchers de tourbillons à la sortie de l'aspirateur et qui provoquent des pulsations de pression qui, elles, sont parfois d'un ordre de grandeur absolument pas négligeable dans les problèmes de cavitation.

D’autre part, l'influence de l'échelle sur le comportement des couches limites est bien connu pour son effet sur les rendements et les puissances des machines; on a même pu élaborer des formules de correction qui permettent assez bien d'en tenir comple. Il est évidemment possible et souhaitable d'appliquer les mêmes considérations à l'étude des sigma criliques. Des calculs très simples montrent que surtout si l'amélioration des rendements se localise dans l'aspirateur, la propension a la cavitation peut être beaucoup plus grande sur In machine prototype que sur le modèle.

Sur le deuxième point: similitude de Froude, nous pensons également que le professeur 'Thoma doive être suivi, bien que, dans certains cas, et sous réserve de certaines précautions, on puisse parfois s'écarter plus ou moins de la similitude de Froude. 


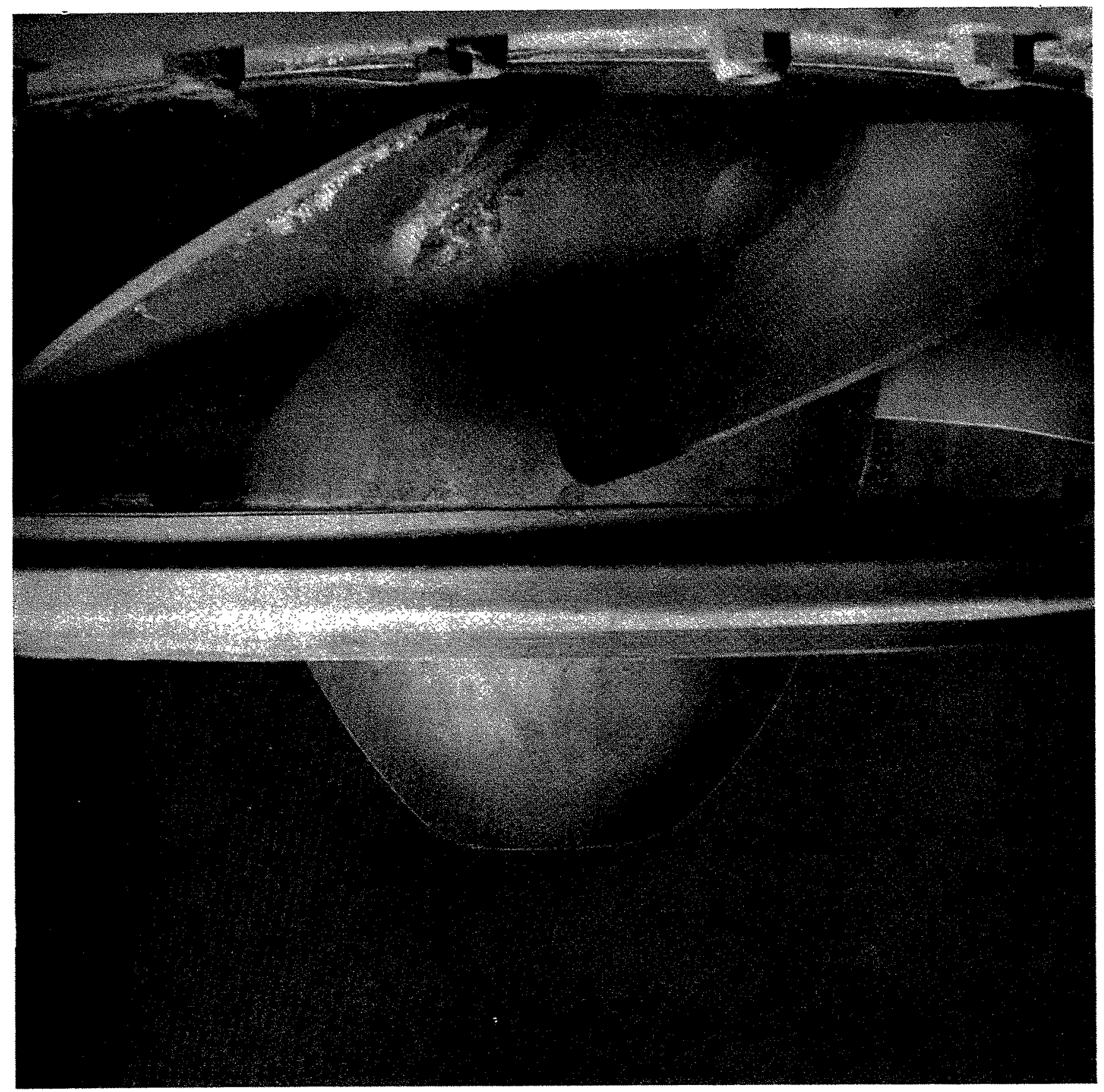

Fxa. 1. - Turbine Kaplan rapide.

Aspect de la cavitation pour la valeur critique des $T$ (début de chute de puissance et de rendement).

Ce problème, ou plutôt les problèmes que pose l'emploi simultané des lois de Froude et de Thoma, ont été posés avec beaucoup de clarté par M. le professeur Gerber.

On sait que la nécessité de combiner l'égalité des sigma avec celles des nombres de Froude se justifie très simplement par la nécessité de réduire dans la même proportion les dépressions statiques résultant des différences d'altitude et les dépressions dynamiques résultant des vitesses d'écoulement de l'eau. Il est évident que les différences de pression statique pourront être négligées et donc le nombre de Froude si les différences d'altitude au sein de la machine étudiée deviennent faibles devant les dépressions dynamiques. Ceci ne veut pas dire cependant que pour une machine ayant un faible développement en hauteur, on puisse toujours s'écarter très librement de la similitude de Froude. Un calcul simple, imaginé par mon collaborateur M. Ginaud, permet de calculer l'erreur maximum que l'on risque de commettre 
sur l'implantation d'une machine en s'écartant de la similitude de Froude.

En appelant:

$\Delta h_{s}$ : l'erreur maximum possible sur le calage industriel;

$l:$ la hauteur totale de la roue;

y. : l'échelle des chutes;

0 : l'échelle des dimensions.

Si $\mu .<\theta \quad \Delta h_{s}=1\left(1-\frac{\mu}{\eta}\right)$

Si $\mu>\theta \Delta h_{s}=1\left(\frac{\mu}{0}-1\right)$

Les expériences décrites par MM. Cox et Craybra dans la communication no 12 nous apportent une confirmation assez pittoresque de la nécessité de la similitude de Froude, dans certaines expériences de cavitation.

Etudiant la poche emplie d'air et de vapeur qui, pour certains sigma se forme dans le sillage d'un obstacle circulaire, les auteurs ont mis en évidence dans certaines circonstances lexistence de deux tourbillons prolongeant la poche vers l'aval et entraînant de l'air on de la vapeur (fig. 2), puis ils ont pu expliquer l'exis-

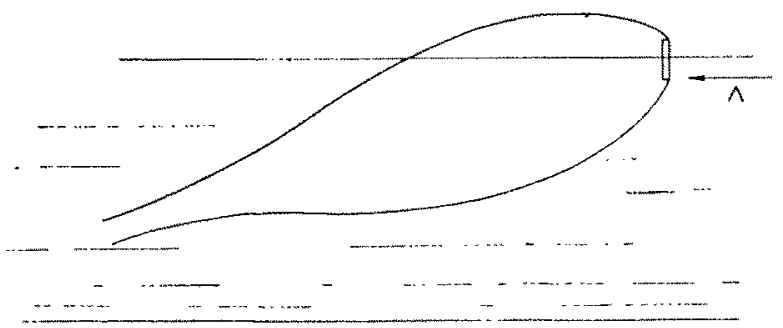

Fla. 2. -- Expériences de R. N. Cox at Vr. A. Clayden. bines.

M. GERBEr, entre autre, a étudié en s'appliquant plus particulièrement aux problèmes des tur-

Pour les turbines Kaplan de basse chute, la similitude de Froude conduit forcément à des chutes d'essais relativement faibles et par conséquent, comme le remarque M. Gerber, à des puissances minimes. A notre avis, et nous sommes certains que M. Gerrer a pensé également à cet aspect du problème, l'adoption d'échelles trop réduites pour le modele et pour la chute peul aussi avoir des conséquences funestes au point de vue des nombres de Reynolds. En dehors de l'effet graduel et certainement modéré du nombre de Reynolds sur les couches limites et les fluctuations turbulentes, il existe un autre effet beaucoup plus grave et que tous les turbiniers ont constaté : si l'on descend en dessous d'un certain nombre de Reynolds critique, les rendements s'effondrent et le régime devient tout à fait inslable, avec éventuellement des doubles régimes très éloignés l'un de l'autre. Il est donc indispensable de se tenir assez largement au-dessus de ces nombres de Reynolds critiques; ce qui oblige, si l'on veut utiliser la similitude de Froude pour des turbines Kaplan de basses chutes, à adopler des modèles de grandes dimensions. Les puissances, malgré tout, restenl relativement peu blevées

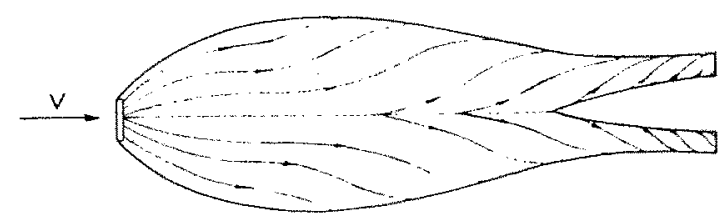

tence de ces tourbillons et en calculer les caracléristiques essentielles; en élaborant une théorie dont le point de départ est la circulation engendrée autour de la poche par la différence d'altitude entre la partie supérieure et la partie inférieure de cette poche. Il est vraiment remarquable d'avoir pu donner une thérie et un calcul assez exacts de ce phénomène curieux et il est surprenant par ailleurs qu'une cause aussi minime apparemment que la hauteur de la bulle puisse être à l'origine de ces deux. tourbillons très caractéristiques.

Reconnaître l'opportunité de réaliser à la fois la similitude de Thoma et celle de Froude nous amène à examiner les conséquences de ces lois au point de vue de la technique expérimentale et au point de vue des autres phénomènes que cela nous oblige à négliger. C'est ce que et il est nécessaire de prendre beaucoup de pricautions si l'on veut les mesurer avec précision.

Une autre difficulté liée à l'utilisation de la similitude de Froude, combinée à celle de Thoma, tient au fait qu'une grande partic de la machine essayée se trouve soumise à un vide assez poussé ce qui fait surgir deux problèmes dont M. Gerber nous a entretenus.

Le premier concerne la nécessité d'éviter les entrées d'air extérieures. Cest un problème purement technologique, que pour notre part nous avons résolu par l'adoption de joints soignés et la disposition de joints noyés autour de l'arbre et des directrices.

L'autre problème est lié à la nécessité de maintenir une certaine quantité d'air dissous 
dans l'eau pour éviter, comme nous l'avons dit plus haut, le retard à la cavitation.

Cet air dissous, si les pressions sont partoul très basses, aura vite fait de transformer l'eau en une émulsion opaque qui, d'une part modifera considérablement les conditions de fonctionnement de la machine et d'autre part, rendra impossible la visualisation et la photographie. Fort heureusement pour les laboratoires d'essais, le résorbeur à été inventé et mis au point par le professeur Knapp; lapplication que nous en avons faite dans notre nouveau laboratoire de cavitation mis en route en 1952 s'est avérée entièrement efficace.

Les temps dont les bulles disposent pour se dilater, puis se résorber au sein de la turbine, ne sont évidemment pas «à l'échelle», mais ceci ne nous semble pas grave car les études, dont plusieurs ont fait l'objet des communicalions à Londres, sur la vie des bulles de vapeur montrent que cette vie est extrêmement brève : le processus de dilatation et de collapsus se passe dans les temps de l'ordre du millième de seconde. Il semble bien, dans ces conditions, que les bulles, aient, même dans le modèle réduit, le temps de vivre leur vie, comme elles le font dans la machine industrielle. L'emploi de la similitude de Froude avec des modeles de grandes dimensions, est d'ailleurs plus favorable à ce point de vue que celle qui correspond à l'utilisation de modèle de faibles dimensions sous chutes élevées. Les temps de parcours, en effet, sont inversement proportionnels à la chute d'essais et proportionnels aux dimensions du modèle.

\section{CONCLUSION}

Pour conclure, nous voudrions dire que si l'expérimentation sur modèle réduit de cavitation ne permet pas de représenter tout ce qui se passe dans la réalité au début de la cavitation, on ne dispose pas non plus d'observations directes sur les turbines industrielles.

On lira avec beaucoup d'intérêt le compterendu des observations faites par A. F. WeEks sur des hélices de bateaux. Il serait très intéressant de se livrer à des observations de même genre sur certaines turbines industrielles et de comparer ce qu'on aurait pu voir ou photographier avec ce que montre le modèle réduit.

Le modèle réduit, entre autres choses auxquelles il doit renoncer, ne peut guère nous renseigner directement sur l'amplitude et les fréquences des vibrations imposées à la turbine. Comme le remarque $M$. Ziegler, le caractère pulsatoire des poches de cavitation, tel qu'il est décrit par M. Knapp, pourrait bien être à l'origine de certains phénomènes de vibrations et de fatigue observés dans les turbines industrielles. On n'en est certainement pas encore à formuler les lois de similitude de ces phénomènes.

Je ne crois pas qu'on soit prêt, non plus, de codifier la similitude de l'érosion. Il intervient certainement dans ce problème en dehors de facteurs physiques assez nombreux, des variables plus ou moins aléatoires parmi lesquelles il fant ranger:
- Les écarts locaux entre le tracé idéal des aubes et leur tracé réel;

- La courbe d'exploitation de la machine : non seulement les charges qu'on fait subir à la turbine et leur durée relative interviennent, mais encore, au moins dans certains cas, la façon de les faire succéder.

La connaissance insuffisante des phénomènes de cavitation et des lois qui les régissent, devrait, à notre avis, conduire à une très grande prudence en matière de calage, car si on voit très facilement l'économie que peut procurer une implantation plus élevée des machines, on voit moins bien les conséquences possibles sur l'exploitation ultérieure. Une perte non prévue de rendement, même minime, peut faire payer très cher la pseudo-économie réalisée sur les frais d'installation. Elle risque fort d'ailleurs de s'accompagner de frais d'entretien énormes.

Les connaissances sur la cavitation, même si on tient compte de leurs progrès récents et du développement des moyens d'expérimentation, sont encore trop faibles à notre avis pour qu'on puisse prétendre se placer toujours à l'extrême limite sous prétexte d'optimum économique ou plutôt on devrait faire intervenir dans la définition de cet optimum économique une notion de risque, dont l'origine est dans l'ignorance relative où nous sommes. 


\section{DISCUSSION}

président: M. Galutes.

M. le Président souligne l'intérêt du jugement personnel de M. Duront sur l'ensemble des expériences et des considerations curieuses qui ont été présentées à Londres a loccasion du symposium « Cavitations».

M. Bencenox signale l'intérêt que peut présenter pour la critique de la eavitation l'examen des caractéristiques des pompes qui sont affectées avee netteté par ce phénomène.

Pour les pompes à faible vitesse spécifique, pour lesquelles les canaux formés par les aubes sont très longs et où, dans des fonctionnements normaux, les conditions à la sortie ne sont pratiquement pas influencées par des tracés différents de l'œillard, l'altération de la cáractéristique hauteur en fonction des débits, due à la eavitation, se traduit par une chute verticale sans zone de transition, e'est-á-dire sans raccordement par un congé de la verticale à la caractéristique normale.

En rognant progressivement la roue, ce qui réduit la longueur des canaux, il apparaît un congé, la chute verticale (cavitation globale) se produisant cependant toujours pour le même débit.

Ceci met en évidence qu'avant d'atteindre ln cavitation globale il se produit des phénomènes secondaires dont, certainement entre autres, le dégagement des gaz dissous, qui n'altèrent pas la caractéristique lorsque les canaux sont très longs, et qui ne commencent ì être décelés par l'examen de ces caractéristiques que lorsque les canaux sont réduits en longueur.

Pour la même raison, lorsque les vitesses spécifiques croissent, comme la longueur des canaux est de plus en plus courte, la zone de transition (raccordement de la caractéristique normale à la verticale de cavitation globale) devient de plus en plus importante. Pour les grandes vitesses spécifiques, ces caractéristiques hauteursdébits sont même affectées jusqu'à débit nul, c'est-à-dire qu'en fonction de la hauteur d'aspiration, la caractéristique hauteur est altérée partout.
On peut donc dire que les phénomènes pcu connus qui précèdent la cavitation globale sont décelés par les essais sur les pompes à grande vitesse spécifique, mais non sur les pompes à faible $n_{\mathrm{s}}$ où cependant ils doivent exister egalement.

Ceci montre qu'il faut etre très prudent dans l'extrapolation par similitude des résultats d'essais, faits dans certaines conditions, it d'autres applications.

D'autre part, M. Bencenon s'inquiete de voir appliquer la similitude de Froude, car pour le dégagement des gaz dissous, qui doit avoir une importance non nëgligeable, il faudrait réaliser l'égalité des pressions entre le modèle et la machine.

M. Dupont pense que M. Bergeron soulève la un probblème délicat : en réalité, la similitude de FrondeThomas s'applique non aux pressions mais à la différence entre la pression locale et la tension de vapeur. Or, il est bien difficile, malheureusement, de réduire la tension de vapeur à l'échelle, quand on opère avec de l'eau.

M. Bengenon ajoute que, d'après un film récent du Laboratoire de l'Ecole des Arts et Métiers de Paris, In pulsation dans la cavitation est de lordre du $1 / 100^{\circ}$ de seconde et pent d'aillenes se caleuler.

D'après un certain nombre de mesures de pression in situ dans des aspirateur's de turbines, M. Willm a observé des phénomènes pulsatoires très marqués de l'ordre de la seconde : dans certains aspirateurs il y a simplement un bruit de fond maís dans d'autres, le régime pulsatoire est de très grande amplitude: il peut atteindre $17 \mathrm{~m}$. pour une chute de 110 à 120 mètres. Il y' a interaction entre la cavitation et ces phénomènes pulsatoires.

M. FERTY pense que pour résumer, on peut dire que nous nous préoccupons de donner un caractère objectif aux phénomènes vibratoires et de ne pas les caractériser simplement par des bruits.

La seance est levée à 12 h. 35 .

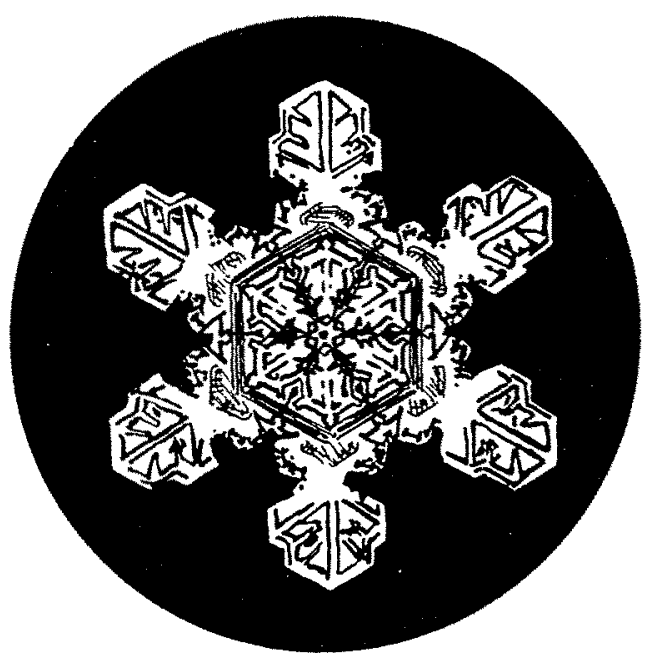

